\title{
Velocity dispersion in GaN-based surface acoustic wave filters on (0001) sapphire substrates
}

\author{
Naoteru Shigekawa $^{1 a)}$, Kazumi Nishimura ${ }^{1}$, Haruki Yokoyama ${ }^{1}$, \\ Kenji Shiojima $^{1}$, and Kohji Hohkawa ${ }^{2}$ \\ ${ }^{1}$ Photonics Laboratories, Nippon Telegraph and Telephone Corporation \\ Morinosato-Wakamiya 3-1, Atsugi, Kanagawa 243-0198, Japan \\ ${ }^{2}$ Department of Engineering, Kanagawa Institute of Technology \\ Atsugi, Kanagawa 243-0292, Japan \\ a)shige@aecl.ntt.co.jp
}

Abstract: We fabricated surface acoustic wave (SAW) filters on three nominally $2-\mu$ m-thick undoped GaN layers grown on (0001) sapphire substrates. We extracted SAW velocities for frequencies of the central and subsidiary peaks in the $\left|\mathrm{S}_{21}\right|$ spectra by applying the $\delta$ function model. We further obtained thicknesses of respective GaN layers by analysing their reflectivity spectra, and established the SAW velocity dispersion. The respective dispersion data were found to lie close to each other, which suggests that characteristics of GaN-based SAW devices can be designed with improved accuracy.

Keywords: GaN, sapphire, surface acoustic wave, SAW, velocity dispersion

Classification: New functional devices and materials

\section{References}

[1] O. Ambacher, "Growth and applications of Group III nitrides," J. Phys. D: Appl. Phys., vol. 31, no. 20, pp. 2653-2710, Oct. 1998.

[2] K. Shiojima, T. Makimura, T. Kosugi, S. Sugitani, N. Shigekawa, H. Ishikawa, and T. Egawa, "High-power AlGaN/GaN dual-gate high electron mobility transistor mixers on SiC substrates," Electron. Lett., vol. 40, no. 12, pp. 775-776, June 2004.

[3] T. Kikkawa, T. Maniwa, H. Hayashi, M. Kanamura, S. Yokokawa, M. Nishi, N. Adachi, M. Yokoyama, Y. Tateno, and K. Joshin, "An Over 200-W Output Power GaN HEMT Push-Pull Amplifier with High Reliability," 2004 IEEE MTT-S Int. Microwave Symp. Digest, pp. 1347-1350, IEEE, Piscataway, NJ, USA, 2004.

[4] M. Nishijima, T. Murata, Y. Hirose, M. Hikita, N. Negoro, H. Sakai, Y. Uemoto, K. Inoue, T. Tanaka, and D. Ueda, "A K-Band AlGaN/GaN HFET MMIC Amplifier on Sapphire Using Novel Superlattice Cap Layer," 2005 IEEE MTT-S Int. Microwave Symp. Digest, TU4B-6, IEEE, Piscataway, NJ, USA, 2005.

[5] C. Deger, E. Born, H. Angerer, O. Ambacher, M. Stutzmann, J. Hornsteiner, E. Riha, and G. Fischerauer, "Sound velocity in $\mathrm{Al}_{\mathrm{x}} \mathrm{Ga}_{1-\mathrm{x}} \mathrm{N}$ 
thin films obtained by surface acoustic-wave measurements," Appl. Phys. Lett., vol. 72, no. 19, pp. 2400-2402, May 1998.

[6] S.-H. Lee, H.-H. Jeong, S.-B. Bae, H.-C. Choi, J.-H. Lee, and Y.-H. Lee, "Epitaxially Grown GaN Thin-Film SAW Filter with High Velocity and Low Insertion Loss," IEEE Trans. Electron Devices, vol. 48, no. 3, pp. 524-529, March 2001.

[7] K. H. Choi, H. J. Kim, S. J. Chung, J. Y. Kim, T. K. Lee, and Y. J. Kim, "Experimental and theoretical characterization of the surface acoustic wave propagation properties of GaN epitaxial layers on c-plane sapphire," J. Mater. Res., vol. 18, no. 5, pp. 1157-1161, May 2003.

[8] K. Nishimura, N. Shigekawa, H. Yokoyama, and K. Hohkawa, "Temperature Dependence of Surface Acoustic Wave Characteristics of GaN Layers on Sapphire Substrates," Jpn. J. Appl. Phys., vol. 44, no. 18, pp. L564-L565, April 2005.

[9] K. Hohkawa, C. Kaneshiro, K. Koh, K. Nishimura, and N. Shigekawa, "Study on Photo-induced Acoustic Charge Transport Effect in GaN Film," 2005 IEEE MTT-S Int. Microwave Symp. Digest, WE1B-3, IEEE, Piscataway, NJ, USA, 2005.

[10] N. Shigekawa, K. Nishimura, H. Yokoyama, and K. Hohkawa, "Side-gate effects on transfer characteristics in GaN-based transversal filters," Appl. Phys. Lett., vol. 87, no. 8, 084102, Aug. 2005.

[11] K. Shiojima and N. Shigekawa, "Thermal Stability of Sheet Resistance in AlGaN/GaN 2DEG Structure," Phys. Stat. Sol. (c), vol. 0, no. 1, pp. 397-400, Dec. 2002.

[12] R. H. Tancrell and M. G. Holland, "Acoustic Surface Wave Filters," Proc. IEEE, vol. 59, no. 3, pp. 393-409, March 1971.

\section{Introduction}

(Al)GaN layers grown on (0001) sapphire or semiinsulating $\mathrm{SiC}$ substrates have been widely investigated for high-power, high-frequency electron devices due to their excellent electrical and thermal properties [1]. AlGaN/GaN field-effect transistors have been successfully applied to dual-gate mixers [2] as well as to L- or K-band power amplifiers [3, 4]. Recently, (Al)GaN-based surface acoustic wave (SAW) devices have been investigated for the purpose of exploiting both the marked piezoelectric properties and semiconducting characteristics of group-III nitrides $[1,5,6,7,8]$. Change in filter characteristics due to ultraviolet illumination [9] and that due to DC bias voltages [10] have also been examined.

The SAW velocities in GaN-on-sapphire systems, which are proportional to centre frequencies of SAW filters, are sensitive to the GaN-layer thickness because elastic properties in GaN are different from those in sapphire (velocity dispersion) [5, 7]. Furthermore, in general, there remains a run-to-run variation of several percents in actual thicknesses of epitaxially-grown GaN layers with the fixed nominal thicknesses. The uncertainty in the GaN-layer thickness could be expanded when the GaN layers are dry-etched because of probable fluctuation in the etching rate. In order to design characteristics of the GaN-based SAW devices, consequently, we need to accurately measure 
the thickness of GaN layers and the SAW velocity dispersion characteristics.

In this letter, we extract the SAW velocities from RF characteristics of SAW filters fabricated on different GaN layers with the same nominal thickness. Furthermore, by locally measuring thickness of GaN layers using optical equipments [11], we construct the SAW velocity dispersions in the respective GaN-on-sapphire systems and discuss their descripancies with one another.

\section{SAW filter fabrication and characterisation}

We prepared three undoped GaN layers on (0001) sapphire substrates by metal organic chemical vapour deposition. The samples are hereafter referred to as A, B, and C. Their nominal GaN layer thickness is commonly $2 \mu \mathrm{m}$. In a preparatory study, we measured sheet resistance of the three samples by characterising four-terminal devices with $\mathrm{Ti} / \mathrm{Al} \mathrm{Ohmic} \mathrm{contacts.} \mathrm{The} \mathrm{sheet}$ resistances of $\mathrm{C}$ was $\approx 45 \mathrm{k} \Omega$. The capacitance of $\mathrm{Al}$ Schottky contacts on $\mathrm{C}$ was found to be $80 \sim 90 \mathrm{pF} / \mathrm{mm}^{2}$. These results indicate [10] that there is formed in $\mathrm{C}$ a depletion region with thickness of $\sim 1 \mu \mathrm{m}$, below which a conductive neutral region is located. No significant results were obtained for the other two layers, which suggests that their resistivities are markedly high and their residual impurity concentrations are unmeasurably low.

We fabricated interdigital transducers (IDTs) in the normal geometry on the respective GaN surfaces and formed SAW filters. The width and separation of the IDTs are $2 \mu \mathrm{m}$. The number of IDT pairs in each transducer is 50. Two transducers in a single SAW filter are separated along the $\{1 \overline{1} 00\}$ direction of sapphire substrates. We simultaneously formed SAW filters with IDT width and separation of $0.8 \mu \mathrm{m}$. Details of the filter fabrication process were previously reported [8].

We measured RF characteristics of the SAW filters at room temperature. The incident RF power was $20 \mathrm{dBm}$. We confirmed that difference in the surface temperatures of devices was less than 5 degrees in measurements. Achieved $\left|\mathrm{S}_{21}\right|$ spectra for the three 2- $\mu \mathrm{m}$ SAW filters are shown in Figs. 1 (a), (b), and (c). A central peak and side lobes are clearly observed in each spectrum. The first subsidiary peaks are lower than the central peak by $26 \mathrm{~dB}$ in each spectrum. The centre frequencies are $581.1,586.0$, and $589.3 \mathrm{MHz}$ in devices on $\mathrm{A}, \mathrm{B}$, and $\mathrm{C}$, respectively. Their relative deviation is as large as $1.4 \%$ although the three devices were fabricated on GaN layers with the same nominal thickness. Similar difference in centre frequencies was also observed in $\left|\mathrm{S}_{21}\right|$ spectra for $0.8-\mu \mathrm{m}$ devices (not shown). It is also found that heights of the central peaks are $-27,-29$, and $-37 \mathrm{~dB}$ in devices on $\mathrm{A}, \mathrm{B}$, and $\mathrm{C}$, respectively. Lower peak heights observed for devices on $\mathrm{C}$ are assumed to be related to its smaller resistivity since SAW filters on counter-doped GaN layers with higher resistivities reportedly reveal larger $\left|\mathrm{S}_{21}\right|$ values [6].

Using a microscope-based optical equipment, we focused white light at several points around the investigated SAW filters in the respective samples, and measured reflectivity spectra there. Obtained reflectivity spectrum at a point on B is shown in Fig. 2 as an example. The thickness in each GaN 

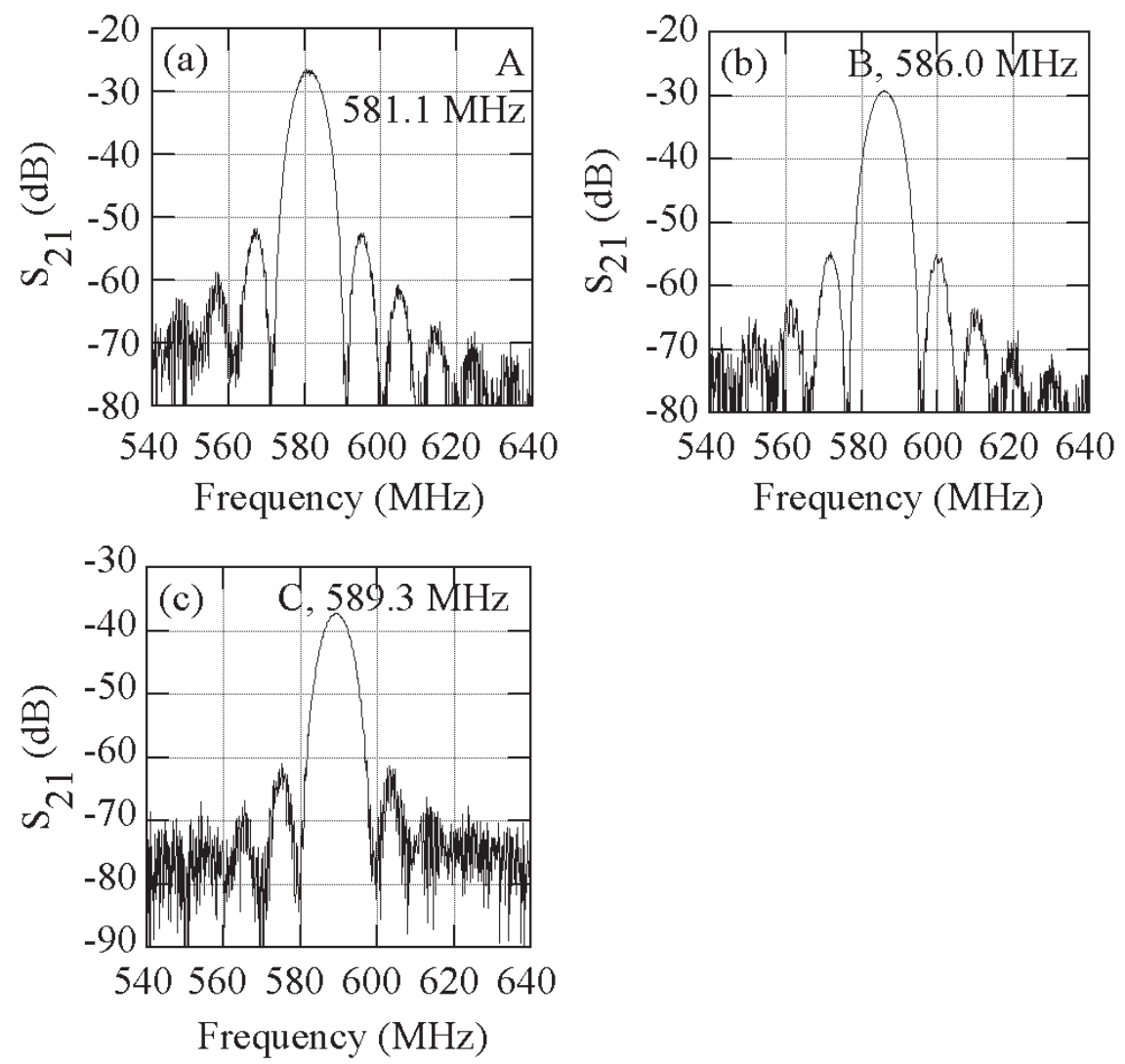

Fig. 1. Frequency dependencies of $\left|\mathrm{S}_{21}\right|$ in 2- $\mu \mathrm{m}$ SAW filters fabricated on A to C. The centre frequencies are also shown for the respective spectra.

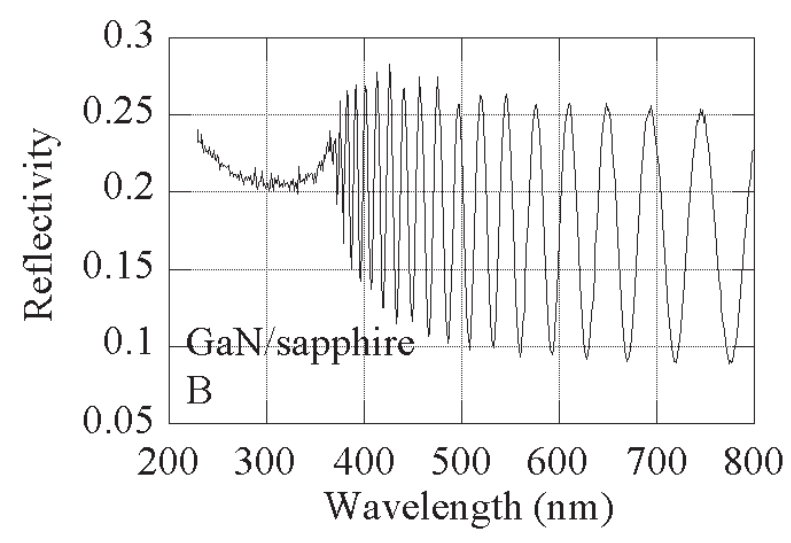

Fig. 2. Reflectivity spectrum measured at a point close to investigated SAW devices on B.

layer $h$, which was deduced by fitting each spectrum and averaging, are 2.056, 1.955, and $1.922 \mu \mathrm{m}$ in A, B, and C, respectively. Their standard deviations are typically $\approx 0.01 \mu \mathrm{m}$. 


\section{SAW velocity dispersion}

Noting that the difference in heights between the central and the first subsidiary peaks is $26 \mathrm{~dB}$, we assume that the SAW filter characteristics can be described using the simplest $\delta$-function model [12]. In this model, magnitude of the frequency response of filters is expressed as

$$
\left|\mathrm{S}_{21}(\omega)\right| \propto\left|\frac{\sin \left(\frac{N \omega d}{v_{\mathrm{SAW}}}\right)}{\cos \left(\frac{1}{2} \frac{\omega d}{v_{\mathrm{SAW}}}\right)} \cos \left(\frac{1}{4} \frac{\omega d}{v_{\mathrm{SAW}}}\right)\right|^{2},
$$

where $v_{\mathrm{SAW}}$ is the SAW velocity, $\omega$ the angular velocity, $d$ the sum of the width and separation of IDT's, and $N$ the number of IDT pairs (50). We determined $(\omega d) / v_{\text {SAW }}$ values giving the central and subsidiary peaks from the above equation, and defined $v_{\text {SAW }}$ by referring to the frequency at each peak in the measured $\left|S_{21}\right|$ spectra. We further determined the SAW wavelength at the respective peaks $(\lambda)$ by $\lambda=2 \pi v_{\mathrm{SAW}} / \omega$.

Extracted $v_{\text {SAW }}$ and $\lambda$ for the $2-\mu \mathrm{m}$ and $0.8-\mu \mathrm{m}$ devices are shown in Figs. 3 (a) and (b) in a fashion of relationships between $v_{\mathrm{SAW}}$ and product of $h$ and SAW wavenumber $k(=2 \pi / \lambda$.) A solid curve is shown in each figure as an eyeguide. The maximum deviations among the data points, which are denoted by arrows, are approximately $30 \mathrm{~m} / \mathrm{s}(0.7 \%)$ and $20 \mathrm{~m} / \mathrm{s}(0.5 \%)$ in the $2-\mu \mathrm{m}$ and $0.8-\mu \mathrm{m}$ devices, respectively.

Errors bars of the respective data are due to the standard deviation in $h$ for the horizontal direction and uncertainties in frequencies at the central or subsidiary peaks in the $\left|\mathrm{S}_{21}\right|$ spectra for the vertical direction, respectively. Given that the temperature coefficient of frequency, or the ratio of variation of the centre frequency to ambient temperature change, is 45 and $30 \mathrm{ppm} / \mathrm{deg}$. for $h k \approx 1.6(h=2 \mu \mathrm{m}$ and $\lambda=8 \mu \mathrm{m})$ and $3.9(h=2 \mu \mathrm{m}$ and
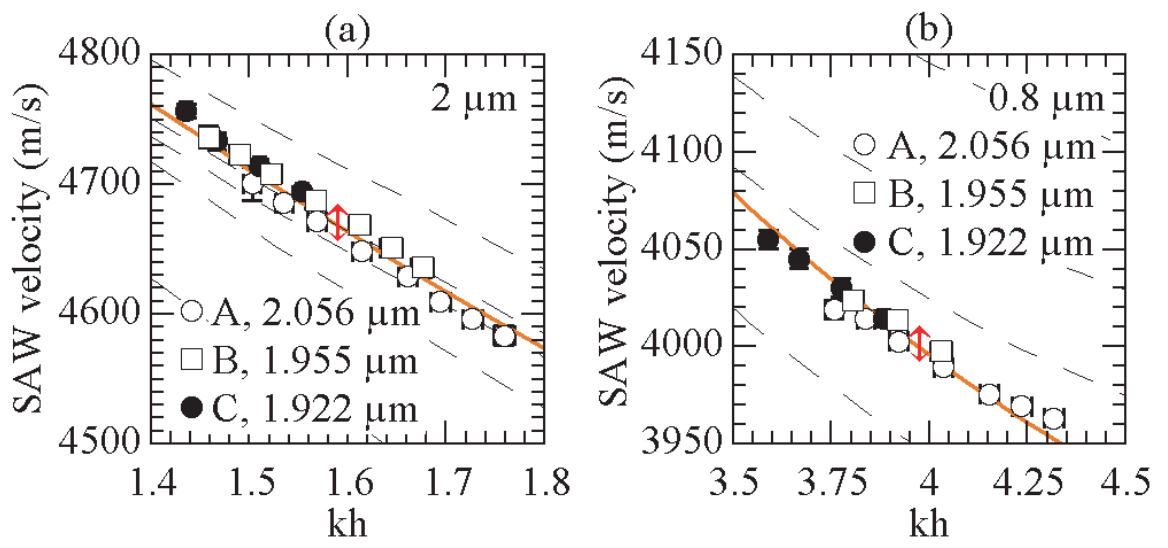

Fig. 3. Relationships between the SAW velocity and the GaN-layer-thickness-wavenumber product for the $2-\mu \mathrm{m}$ (a) and 0.8- $\mu \mathrm{m}$ (b) devices. Curves for eyeguide and velocity dispersions obtained by calculations are also shown by solid and dashed lines, respectively. Measures of the maximum descripancies among the data are also shown. 
$\lambda=3.2 \mu \mathrm{m}$ ) [8], possible influence of uncertainty in ambient temperatures, 5 degrees at maximum, on $v_{\text {SAW }}$ is likely to be negligibly small.

Dashed lines show velocity dispersion curves obtained by calculation using different elastic modulae for GaN [7]. It is found that these curves are largely separated from one another, and the obtained data points lie among these curves, which suggests that elastic modulae for GaN could be optimised for reproducing the measured data.

The fact that all the velocity dispersion data lie close to one another indicates that a "universal curve" describing the velocity dispersion can be established. More importantly, optical methods like that employed in the present work are likely to be useful in order to quantitatively grasp the GaNlayer thickness. This means that specified thickness of GaN layer and hence specified SAW velocity are achievable by, for example, combining dry etching and layer-thickness measurements. The obtained result, consequently, suggests that the characteristics of GaN-based SAW devices can be designed with improved accuracy.

Given that the velocity dispersions of the three samples approximately agree with one another, the SAW velocity is assumed not to be sensitive to residual impurity concentrations or resistivities in GaN layers. This view is consistent with our finding that the centre frequencies of SAW filters on a conductive GaN layer are almost independent of side-gate bias voltages [10]. More precise studies such as group-delay analyses, however, are required in order to clarify the influence of the electrical properties of GaN layers on SAW transport characteristics.

\section{Conclusion}

We fabricated surface acoustic wave (SAW) filters on three GaN-on-sapphire systems with nominally $2-\mu \mathrm{m}$ thick GaN layers. We deduced SAW velocity dispersion by combining SAW velocities and thicknesses of the respective GaN layers, which were obtained by analysing RF characteristics of filters and reflectivity spectra, respectively. All the velocity dispersion data were found to lie close to a single curve although the centre frequencies of the respective filters are markedly different from one another, The result means that characteristics of GaN-based SAW devices can be designed with improved accuracy.

\section{Acknowledgements}

The authors are grateful to Takashi Makimura for his advice in device fabrication, and to Takashi Kobayashi and Takatomo Enoki for their encouragements. 